\title{
REABILITAÇÃO EM DISFAGIA OROFARÍNGEA NEUROGÊNICA: SABOR AZEDO E TEMPERATURA FRIA
}

\author{
Rehabilitation in neurogenic oropharyngeal dysphagia: \\ sour taste and cold temperature
}

\author{
Paula Cristina Cola ${ }^{(1)}$, Ana Rita Gatto (2), Roberta Gonçalves da Silva ${ }^{(3)}$, \\ Arthur Oscar Schelp ${ }^{(4)}$, Maria Aparecida Coelho de Arruda Henry ${ }^{(5)}$
}

\section{RESUMO}

Tema: reabilitação em disfagia orofaríngea neurogênica Objetivo: apresentar revisão de literatura sobre os controles neurofisiológicos da deglutição orofaríngea e a influência do sabor azedo e da temperatura fria no mecanismo da deglutição. Conclusão: quanto à questão do controle central da deglutição, ainda existem controvérsias em relação ao sabor azedo e a temperatura fria. Esses dois parâmetros provocam mudanças na dinâmica da deglutição, podendo trazer benefícios aos indivíduos acometidos por disfagia orofaríngea neurogênica. Porém, tais achados sugerem a necessidade de investigações futuras com populações randomizadas.

DESCRITORES: Transtornos de Deglutição; Reabilitação; Acidente Cerebral Vascular

\section{INTRODUÇÃO}

As dificuldades de deglutição são comuns em pacientes após acidente vascular encefálico (AVE). Estudos diversos relataram incidência de disfagia

(1) Fonoaudióloga Assistente de Pesquisa do Ambulatório de Disfagia da Universidade Estadual Paulista em Marília SP; Doutoranda em Bases Gerais da Cirurgia pela Faculdade de Medicina de Botucatu da Universidade Estadual Paulista - SP.

(2) Fonoaudióloga do Hospital das Clínicas da Universidade Estadual Paulista de Botucatu - SP; Especialização em Motricidade Oral. Mestranda em Bases Gerais da Cirurgia pela Faculdade de Medicina de Botucatu da Universidade Estadual Paulista - SP.

(3) Fonoaudióloga; Professora do Departamento de Fonoaudiologia da Universidade Estadual Paulista em Marília SP; Doutora em Fisiopatologia em Clínica Médica - Área de Metabolismo e Nutrição pela Faculdade de Medicina de Botucatu da Universidade Estadual Paulista - SP.

(4) Médico Neurologista; Professor do Departamento de Neurologia e Psiquiatria- Faculdade de Medicina de Botucatu da Universidade Estadual Paulista - SP; Doutor em Patologia pela Faculdade de Medicina de Botucatu da Universidade Estadual Paulista - SP.

(5) Médica Gastrocirurgiã; Professora Titular do Departamento de Cirurgia e Ortopedia da Faculdade de Medicina de Botucatu da Universidade Estadual Paulista - SP; Livre-Docente pela Faculdade de Medicina de Botucatu da Universidade Estadual Paulista - SP. orofaríngea que varia de 50 Á $76 \%$ dos pacientes com acidentes vasculares encefálicos mediante avaliação fonoaudiológica clínica ${ }^{1,2}$. A incidência eleva-se para $90 \%$ com avaliação videofluoroscópica da deglutição orofaríngea. A maior sensibilidade do exame videofluoroscópico da deglutição permite detectar tanto as formas mais leves de disfagia orofaríngea, caracterizadas por alterações orais dificilmente visualizadas pela avaliação fonoaudiológica clínica ${ }^{2}$, quanto as alterações faríngeas.

Os distúrbios da deglutição são definidos como disfagia orofaríngea quando apresentam sinais e sintomas específicos, caracterizados por alterações em qualquer fase e/ou entre as etapas da dinâmica da deglutição, podendo ser congênito ou adquirido após comprometimento neurológico, com prejuízo dos aspectos nutricionais, hidratação, função pulmonar e integração social do indivíduo ${ }^{3}$.

A reabilitação na disfagia orofaríngea tem utilizado técnicas fonoaudiológicas que envolvem o sabor azedo e a temperatura fria, com o intuito de aumentar a modulação oral e a resposta faríngea da deglutição ${ }^{3}$.

Este estudo tem como objetivo apresentar revisão de literatura sobre os controles neurofisiológicos da deglutição orofaríngea e a influência do sabor azedo e da temperatura fria no mecanismo da deglutição. 


\section{MÉTODOS}

Para a elaboração deste artigo foi realizada extensa pesquisa bibliográfica em diferentes bases de dados, como Medline, Scielo Brasil, Chile e Espanha e Lilacs, no período de 1920 a 2007 além de livros pesquisados em bibliotecas. Esta pesquisa utilizou os seguintes descritores: disfagia orofaríngea, sabor azedo, temperatura fria, representação cortical e reabilitação. Foram pesquisados no período de 2 anos, março de 2005 a março de 2007, e a análise dos artigos encontrados foi feita baseada nos assuntos como neurofisiologia da deglutição, controle cortical do mecanismo da deglutição e o efeito do sabor e temperatura sobre a resposta faríngea da deglutição.

\section{REVISÃO DE LITERATURA}

O controle neural das fases oral e faríngea da deglutição vem sendo explicado por meio de duas hipóteses a primeira delas é descrita pelo movimento do bolo alimentar através da boca e da faringe, estimulando os receptores sensoriais e desencadeando o próximo passo na seqüência da deglutição. Outra hipótese é que, uma vez a deglutição iniciada, ativa-se um controle programado de uma rede de neurônios no tronco cerebral, centro da deglutição, cuja função é independente de feedback sensorial. E ainda, que o volume do bolo alimentar pode alterar a seqüência da deglutição, modificando, por exemplo, o tempo de abertura do esfíncter cricofaríngeo, mas não interferindo em outras variáveis, como a magnitude da contração faríngea ${ }^{4}$.

Com relação aos receptores sensoriais da cavidade oral, faringe e laringe, estes são inervados por fibras dos nervos trigêmeo (V), facial (VII) e vago (X). O nervo trigêmeo é responsável pelo território da face, lábios, mucosa do vestíbulo, incluindo os dentes, periodonto, palato duro e parte anterior do palato mole, com extensão para porção superior da nasofaringe. O nervo glossofaríngeo e o ramo faringiano do nervo vago são fontes primárias da inervação sensorial da faringe. O nervo vago supre a inervação sensorial da laringe e da epiglote. Em seguida, as fibras aferentes dos nervos cranianos VII, IX e X entram no tronco cerebral a nível medular e seguem ao núcleo do trato solitário. Muitas dessas fibras estão envolvidas na gustação, mas muitas delas têm função mecanoreceptora ${ }^{5}$.

A parte mais ricamente inervada da faringe é a junção na divisão entre a cavidade oral e nasal. Os receptores do epitélio orofaríngeo são predominantemente terminações livres, existindo dois plexos sensoriais na laringe e epiglote, quando compara- dos com um na faringe, sugerindo que a sensibilidade é mais aguda ou mais altamente organizada na laringe ${ }^{5}$.

As aferências na cavidade oral posterior, faringe e laringe são transmitidas para o núcleo do trato solitário e para a córtex cerebral, sendo necessárias para desencadear a resposta faríngea da deglutição na região orofaríngea. Alguns neurônios pré-motores ou interneurônios são encontrados na formação reticular, os quais podem iniciar ou organizar os neurônios motores da deglutição. Esses neurônios são localizados em volta do núcleo do trato solitário e em volta do núcleo ambíguo ${ }^{6}$.

Os receptores são divididos em cinco grupos, de acordo com o tipo de estímulo ao qual eles são mais sensíveis. Os quimioreceptores respondem às moléculas ligantes químicas que se associam ao receptor. Os mecanoreceptores respondem a várias formas de energia mecânica, incluindo pressão, vibração, gravidade, aceleração e som. Os termoreceptores respondem à temperatura, os fotorreceptores, à luz e os nociceptores, a estímulos dolorosos ${ }^{7}$.

Nas últimas décadas surgiram na literatura estudos voltados para a representação cortical do mecanismo da deglutição e com a ajuda de aparelhos de neuroimagem tornou-se possível a melhor compreensão destes aspectos. Alguns estudos mostraram dominância em ambos os hemisférios durante a deglutição de saliva, no entanto, quando a dominância hemisférica foi à direita, a representação na córtex mostrou-se mais intensa. Os estudos mostraram ainda que a ativação cortical e subcortical durante a deglutição é difusa, ocorrendo em vários locais nos lobos frontal, parietal e temporal. Esta ativação é mais específica na córtex motora primária, somatossensorial primária, suplementar, pré-frontal, no giro temporal transverso, no giro cingular, na ínsula, na cápsula interna, nas áreas do discurso, bem como em outras áreas de associação, no giro temporal superior e nas áreas de integração sensório-motora ${ }^{8,9}$.

Já em outro estudo verificou-se que a atividade cerebral foi fortemente lateralizada para o hemisfério esquerdo no córtex sensório-motor durante a deglutição voluntária e menos lateralizada para o hemisfério esquerdo durante a deglutição reflexa. A área de ativação durante a deglutição voluntária foi maior quando comparada à área ativada na deglutição reflexa. Referem ainda que a lateralização encontrada é, principalmente, uma função do sistema motor eferente, enquanto o processamento sensorial, precedido de um ato de deglutir, é representado por ambos hemisférios. Concluem que os achados controversos a outros estudos devem-se, talvez, ao fato de que os estudos encontrados na 
literatura não diferenciam os mecanismos aferentes dos eferentes na dinâmica da deglutição ${ }^{10}$.

Com relação à recepção do sabor a nível periférico, refere-se que o sabor pode desencadear resposta motora da língua e que múltiplas aferências captadas na língua fazem sinapse afetando os motoneurônios do hipoglosso ${ }^{11}$. Já a representação da temperatura na córtex, quando estudada em macacos, foi evidenciada na região orbitofrontal ${ }^{12}$.

Estudos mais recentes investigaram a possível existência de uma representação neuronal para o sabor umami na córtex primária do sabor. Os indivíduos receberam estímulos de sabor doce, salgado, azedo, amargo e umami. Encontraram atividade hemodinâmica na região da ínsula opercular em ambos os hemisférios, com maior ativação no hemisfério direito. Referiram sobre a dificuldade em saber qual região é responsável por cada estímulo separadamente, pois a variação na concentração de determinado sabor também influenciaria ${ }^{13}$.

As questões relacionadas às influências do sabor e da temperatura na dinâmica da deglutição ainda estão longe de elucidação para aplicações clínicas específicas. A definição de critérios, tanto na avaliação quanto na reabilitação de indivíduos disfágicos, ainda apresenta lacunas a serem preenchidas. Os dados de literatura disponíveis permitem antecipar que a modificação do sabor e da temperatura dos alimentos tem influência na dinâmica da deglutição.

Diferentes estudos se propõem a explicar os mecanismos envolvidos na resposta faríngea da deglutição e sua relação com o sabor e a temperatura. Dentre as pesquisas mais antigas sobre as aferências orais para a deglutição, encontramos um trabalho com 126 indivíduos saudáveis, aplicando leves toques, com o uso de uma haste de vidro, em distintas regiões da cavidade oral. $\mathrm{O}$ autor concluiu que a região anterior do pilar das fauces é a mais sensível a este estímulo tátil para desencadear a resposta faríngea da deglutição ${ }^{14}$.

Com relação ao efeito do sabor e da temperatura sobre o mecanismo da deglutição, foram identificados na literatura estudos que incluem indivíduos com alterações neurológicas heterogêneas com registro de mudanças na dinâmica da deglutição quando os fatores sabor e temperatura estão presentes. Tais estudos mostraram mudanças na dinâmica da deglutição. Os autores estudaram 25 indivíduos que apresentavam diagnóstico neurológico variado, como AVE, Doença de Parkinson, Esclerose Múltipla, traumatismo crânio-encefálico, tumor cerebral e paralisia pseudobulbar e analisaram o desencadear da deglutição com aplicação de estímulos na região do pilar das fauces, através do exame de videofluoroscopia. Esses indivíduos, após aplicação de estímulo frio na região do pilar das fauces, apresentaram mudança no desencadear da deglutição, com tempo menor para a resposta faríngea. Concluíram que a sensação térmica fria na região do pilar das fauces intensifica a sensibilidade da área e quando o alimento é apresentado o indivíduo dispara a deglutição e a resposta se torna mais rápida ${ }^{15}$.

Outro estudo em indivíduos com diagnósticos também heterogêneos, paralisia cerebral, traumatismo craniano, acidente vascular encefálico, doença de Alzheimer observou a deglutição de alimentos azedos e doces, separadamente, através do exame de endoscopia de deglutição. Concluíram que o azedo modifica a deglutição, minimizando penetrações e aspirações laringotraqueais em indivíduos com acometimento neurológico. Os autores sugeriram que a mudança na deglutição seja devida ao estímulo azedo aumentar a estimulação trigeminal para o tronco cerebral em indivíduos com distúrbios neurológicos ${ }^{16}$.

Outros estudos relataram que nos indivíduos pós-AVE o tempo de trânsito orofaríngeo é menor, quando o bolo com sabor azedo é deglutido. As vias neuronais do sabor, quando estimuladas com forte sabor, como o azedo, podem servir como estímulo de alerta para o tronco cerebral e centros corticais da deglutição, explicando a mudança significativa nas medidas de tempo em relação às fases da deglutição ${ }^{17}$. Ainda nesta perspectiva, A temperatura fria e o sabor cítrico combinados alteram 0 comportamento da deglutição em indivíduos saudáveis e também nos indivíduos pós-AVE ${ }^{18}$.

Considerando que a maioria destes estudos foram realizados com amostras heterogêneas, doenças neurológicas distintas, deve-se ressaltar as limitações quanto as generalizações. No entanto, atualmente, estudos controlados têm comprovado os resultados positivos sobre a influencia do sabor azedo e da temperatura fria concomitantes no tempo de trânsito faríngeo, com diferença significativa em relação ao sabor e temperatura isolados ${ }^{19}$.

Em estudos experimentais observou-se também que a estimulação na região faringolaríngea com solução azeda facilitou a resposta faríngea da deglutição, sugerindo que essa facilitação pode ser devido ao aumento de aferência via nervo laríngeo superior e nervo glossofaríngeo ${ }^{20,21}$. Além disto, a estimulação elétrica e mecânica fria, evocaram significativamente maior número de deglutições do que a estimulação elétrica sozinha. Os autores concluíram que a ativação dos receptores sensoriais da orofaringe aumenta a freqüência de deglutições quando também ocorre estimulação do nervo laríngeo ${ }^{22}$. 
Novamente quando se fala do efeito da temperatura, encontra-se na literatura a influência da mesma sobre o tempo de trânsito orofaríngeo. Estudo com indivíduos pós-AVE, que apresentavam disfagia orofaríngea, evidenciou que a aplicação térmica (fria) no pilar das fauces com espelho laríngeo, antes do indivíduo deglutir o bolo, levou a um tempo de trânsito orofaríngeo menor que o grupo que não recebeu o referido estímulo ${ }^{23}$. Sugere-se a existência de receptores térmicos no pilar das fauces os quais evocam a deglutição quando estimulados por toques frios e aumentam a resposta faríngea ${ }^{24}$. Em estudo com indivíduos jovens, já saudáveis, a variação da temperatura também evidenciou diferenças sobre os aspectos da deglutição, tanto sensorial como motor ${ }^{25}$.

Quando se utilizou o sabor e a temperatura como estimulação na região do pilar das fauces em indivíduos saudáveis, não ocorreu diferença no tempo para desencadear a resposta faríngea da deglutição comparando os estímulos separadamente. Porém, os estímulos concomitantes, apresentaram tempo menor para desencadear a resposta faríngea da deglutição. Os resultados suportam a explicação que estímulos combinados facilitam o desencadear da resposta faríngea da deglutição ${ }^{26}$. Ainda estudando indivíduos saudáveis observou-se que o início para deglutir é mais rápido com contrações musculares mais fortes quando deglutem o bolo com sabores (doce e azedo) 27,28.

Além de evidências que comprovam o efeito do sabor e da temperatura sobre o desencadear da deglutição, encontra-se na literatura a influência do sabor na pressão da língua sobre o palato duro durante a deglutição. Estudo com eletromiografia de superfície na região da musculatura submental evidenciou pico de pressão da língua significativamente maior com a concentração de açúcar moderada, concentração alta de sal e concentração alta de acido cítrico, comparado com o pico de pressão encontrado com a deglutição de água. Esses resultados sugeriram que as altas concentrações intensificam a aferência do sabor para o núcleo do trato solitário e pode ser crucial na modificação dos comportamentos da deglutição, como na pressão da língua no início da deglutição ${ }^{29}$.
Outros fatores que modificam o mecanismo da deglutição, tanto em indivíduos saudáveis como indivíduos com alterações neurológicas, são as variações na consistência e volume do bolo deglutido, interferindo no tempo de trânsito faríngeo, na força de ejeção como também na abertura do cricofaríngeo ${ }^{30-33}$.

Apesar de diversos estudos apresentarem concordância ao investigarem o efeito das variações dos alimentos (consistência, volume e temperatura) sobre a dinâmica da deglutição, encontramse estudos que não relataram quaisquer modificações, sugerindo que o desencadear da deglutição não é mediado por receptores na região do pilar das fauces ${ }^{34}$. A razão dessa discordância encontra-se, provavelmente, na metodologia aplicada. Essa discordância já começa quando se fala nas fases da deglutição, observando-se várias definições de início e término das fases da deglutição, bem como diversas maneiras como estas são analisadas ${ }^{31,35}$. São necessários investigações futuras envolvendo também indivíduos com acometimento neurológico.

\section{COMENTÁRIOS FINAIS}

Após extensa revisão de literatura, constatouse que o fator térmico frio e o gustativo azedo influenciam na modulação da dinâmica da deglutição, tanto em indivíduos saudáveis como em indivíduos com acometimento neurológico. Poucos estudos investigaram esta questão com uma casuística controlada, não sendo possível a generalização deste impacto para diferentes doenças neurológicas. Com relação ao lado acometido pela lesão e sua correlação com as dificuldades de modulação oral e tempo de trânsito faríngeo, estudos futuros com aparelhos de neuroimagem mais avançados, associados inclusive com videofluoroscopia de deglutição, poderão nos proporcionar maiores esclarecimentos. Todavia, estes estudos sugerem que o sabor azedo e a temperatura fria podem provocar efeito positivo no processo de reabilitação de indivíduos com quadro de disfagia orofaríngea. 


\begin{abstract}
Background: rehabilitation in neurogenic oropharyngeal dysphagia Purpose: to submit an overview of the related literature about neurophisiological control of oropharingeal dysphagia and the role of temperature and sour taste on swallowing mechanisms. Conclusions: as for the neurophysiologic swallowing control, there are still controversies when it comes to sour taste and cold temperature. These two standards can change the swallowing dynamics, and they may bring out benefits to the individuals with neurogenic oropharyngeal dysphagia. Therefore, the findings suggest the need for further investigations with randomized studies.
\end{abstract}

KEYWORDS: Deglutition Disorders; Rehabilitation; Stroke

\section{REFERÊNCIAS}

1. Chen MYM, Ott DJ, Peele VN, Gelfand DW. Oropharynx in patients with cerebrovascular disease: evaluation with videofluoroscopy. Radiol. 1990; 176:641-3.

2. Schelp AO, Cola PC, Gatto AR, Silva RG, Carvalho LR. Incidência de disfagia orofaríngea após acidente vascular encefálico em hospital público de referência. Arq Neuro-Psiquiatr. 2004; 62(2-B):503-6.

3. Furkim AM, Silva RG. Programas de reabilitação em disfagia neurogênica. São Paulo: Frôntis; 1999.

4. Dodds WJ, Stewart ED, Logemann JA. Physiology and radiology of the normal oral and pharyngeal phases of swallowing. Am J Roentgenol. 1990; 154:953-63.

5. Capra NF. Mechanisms of oral sensation. Dysphagia. 1995; 10(4):235-47.

6. Ertekin C, Aydogdu I. Neurophysiology of swallowing. Clin Neurophysiol. 2003; 114:2226-44.

7. Silverthorn DV. Fisiologia humana: uma abordagem integrada. 2. ed. São Paulo: Manole; 2003. p.283-99.

8. Hamdy S, Mikulis DJ, Crawley A, Xue S, Lau $\mathrm{H}$, Henry $\mathrm{S}$, et al. Cortical activation during human volitional swallowing: an event-related fMRI study. Am J Physiol. 1999; 277(1):G219-25.

9. Kern MK, Jaradeh S, Arndorfer RC, Shaker R. Cerebral cortical representation of reflexive and volitional swallowing in humans. Am J Physiol. 2001; 280(3):G354-60.

10. Dziewas R, Soros $P$, Ishii R, Chau W, Henningsen $H$, Ringelstein EB, et al. Neuroimaging evidence for cortical involvement in the preparation and in the act of swallowing. Neuroimage. 2003; 20:135-44.

11. Miller AJ. Oral and pharyngeal reflexes in the mammalian nervous system: their diverse range in complexity and the pivotal role of the tongue. Crit Rev Oral Biol Med. 2002; 13(5):409-25.
12. Kadohisa M, Rolls ET, Verhagen JV. Orbitofrontal cortex: neuronal representation of oral temperature and capsaicin in addition to taste and texture. Neuroscience. 2004; 127(1):207-21.

13. Schoenfeld MA, Neuer G, Tempelmann C, Schübler K, Noesselt T, Hopf JM, et al. Functional magnetic resonance tomography correlates of taste perception in the human primary taste cortex. Neuroscience. 2004; 127(2):347-53.

14. Pommerenke WT. A study of the sensory areas eliciting the swallowing reflex. Am J Physiol. 1928; 84:36-41.

15. Lazzara GI, Lazarus C, Logemann JA. Impact of thermal stimulation on the triggering of the swallowing reflex. Dysphagia. 1986; 1:73-7.

16. Pelletier CA, Lawless HT. Effect of citric acid and citric acid-sucrose mixtures on swallowing in neurogenic oropharyngeal dysphagia. Dysphagia. 2003; 18(4):231-41.

17. Logemann JA, Pauloski BR, Colangelo L, Lazarus C, Fujiu M, Kahrilas PJ. Effects of sour bolus on oropharyngeal swallowing measures in patients with neurogenic dysphagia. J Speech Hear Res. 1995; 38:556-63.

18. Hamdy S, Jilani S, Price V, Parker C, Hall N, Power M. Modulation of human swallowing behavior by thermal and chemical stimulation in health and after brain injury. Neurogastroenterol Motil. 2003; 15(1):69-77.

19. Cola PC, Gatto AR, Spadotto AA, Silva RG, Schelp AO, Henry MACA. Medição do tempo faríngeo da deglutição em indivíduos após acidente vascular encefálico. GED. 2006; 25: 111.

20. Kajii $Y$, Shingai T, Kitagawa J, Takahashi $Y$, Taguchi $Y$, Noda $T$, et al. Sour taste stimulation facilitates reflex swallowing from the pharynx and larynx in the rat. Physiol Behav. 2002; 77:321-5.

21. Kitagawa JI, Shingai T, Takahashi Y, Yamada Y. Pharyngeal branch of the glossopharyngeal nerve plays a major role in reflex swallowing from the 
pharynx. Am J Physiol Regul Integr Comp Physiol. 2002; 282:R1342-7.

22. Chi-Fishman G, Capra NF, McCall GN. Thermomechanical facilitation of swallowing evoked by electrical nerve stimulation in cats. Dysphagia. 1994; 9(3):149-55.

23. Rosenbek JC, Roecker EB, Wood JL, Robbins $\mathrm{J}$. Thermal application reduces the duration of stage transition in dysphagia after stroke. Dysphagia. 1996; 11:225-33.

24. Kaatzke-McDonald MN, Post E, Davis PJ. The effects of cold, touch and chemical stimulation of the anterior faucial pillar on human swallowing. Dysphagia. 1996; 11(3):198-206.

25. Miyaoka Y, Haishima K, Takagi M, Haishima $\mathrm{H}$, Asari J, Yamada $\mathrm{Y}$. Influences of thermal and gustatory characteristics on sensory and motor aspects of swallowing. Dysphagia. 2006; 21(1):38-48.

26. Sciortino KF, Liss JM, Case JL, Gerritsen KGM, Katz RC. Effects of mechanical, cold, gustatory, and combined stimulation to human anterior faucial pillars. Dysphagia. 2003; 18:16-26.

27. Ding R, Logemann JA, Larson CR, Rademaker AW. The effects of taste and consistency on swallow physiology in younger and older healthy individuals: a surface electromyographic study. J Speech Lang Hear Res. 2003; 46(4):977-89

28. Palmer PM, McCulloch TM, Jaffe D, Neel AT. Effects of sour bolus on the intramuscular electromyographic (EMG) activity of muscles in the submental region. Dysphagia. 2005; 20(3):210-7.

29. Pelletier CA, Dhanaraj GE. The effect of taste and palatability on lingual swallowing pressure. Dysphagia. 2006; 21(2):121-8.

30. Cook IJ, Dodds WJ, Dantas RO, Kern MK, Massey BT, Shaker R, et al. Timing of videofluoroscopic, manometric events, and bolus transit during the oral and pharyngeal phases of swallowing. Dysphagia. 1989; 4:8-15.

31. Dantas RO, Kern MK, Massey BT, Dodds WJ, Kahrilas PJ, Brasseur JG, et al. Effect of swallowed bolus variables on oral and pharyngeal phases of swallowing. Am J Physiol. 1990; 258(5):G675-81.

32. Bisch EM, Logemann JA, Rademaker AW, Kahrilas PJ, Lazarus CL. Pharyngeal effects of bolus volume, viscosity and temperature in patients with dysphagia resulting from neurologic impairment and in normal subjects. J Speech Hearing Res. 1994; 37(5):1041-59.

33. Tachimura T, Ojima M, Nohara K, Wada T. Change in palatoglossus muscle activity in relation to swallowing volume during the transition from the oral phase to the pharyngeal phase. Dysphagia. 2005; 20(1):32-9.

34. Kendall KA, Mckenzie S, Leonard RJ, Gonçalves MI, Walker A. Timing of events in normal swallowing: a videofluoroscopic study. Dysphagia. 2000; 15(2):74-83.

35. Kendall KA. Oropharyngeal swallowing variability. Laryngoscope. 2002; 112:547-51.

RECEBIDO EM: 07/07/2007

ACEITO EM: 24/04/2008

Endereço de correspondência:

Av. Salgado Filho, 176

Marília - SP

CEP: $17513-260$

Tel: (14) 3433-4128

E-mail: paccola@ hotmail.com 